ventricular pulsus alternans during acute increase in afterload during balloon pulmonic valvuloplasty. Am J Cardiol 1987; 60:412-3.

13. Gleason WL, Braunwald E. Studies on Starling's law of the heart. VI. Relationship between left ventricular end-diastolic volume and stroke volume in man with observations on the mechanism of pulsus alternans. Circulation 1962;25:841-7.

14. Lab MJ, Lee JA. Changes in intracellular calcium during me chanical alternans in isolated ferret ventricular muscle. Circ Res 1990;66:585-95.

15. Wilson RF, Laughlin DE, Ackell PH, et al. Transluminal, subselective measurement of coronary artery blood flow velocity and vasodilator reserve in man. Circulation 1985;72:8292.

16. Wilson RF, Marcus ML, White CW. Pulmonary inflation reflex: its lack of physiologic significance in coronary circulation of humans. Am J Physiol 1988;255(Heart Circ Physiol 24): H866-71.

17. Wilson RF, White CW. Intracoronary papaverine: an ideal coronary vasodilator for studies of the coronary circulation in conscious humans. Circulation 1986;73:444-51.

\section{Myocardial contrast echocardiography can depict thebesian vein outflow in humans}

Jan H. Cornel, MD, Folkert J. Ten Cate, MD, and Patrick W. Serruys, MD. Rotterdam, The Netherlands

From the Thoraxcenter, Department of Cardiology, Academic Hospital Dijkzigt and Erasmus University Rotterdam.

Supported by grant 85060 from the Netherlands Heart Foundation, 's Gravenhage, The Netherlands.

Reprint requests: Folkert J. Ten Cate, MD, Academic Hospital Dijkzigt, Thoraxcenter Ba 350, Dr. Molewaterplein 40, 3015 GD Rotterdam, The Netherlands.

4/4/35984
Myocardial contrast echocardiography (MCE) has been developed as a method to study myocardial perfusion. ${ }^{1}$ In this report, we describe the ability of MCE to depict thebesian vein outflow in vivo in humans undergoing coronary angiography. The study group consisted of 29 patients undergoing diagnostic coronary arteriography. Twenty-seven patients had coronary artery disease and two had hypertrophic cardiomyopathy. The study protocol was approved by the Thoraxcenter Institutional Review Board. MCE was performed immediately after completion of the coronary arteriography by injecting sonicated iopamidol into the left main coronary artery. ${ }^{2}$ Using twodimensional echocardiography, apical views were obtained of the left ventricular cavity.

Two independent observers reviewed the echocardiographic images frame by frame. The appearance of contrast was sought in the left ventricular cavity subsequent to myocardial opacification. Contrast was noted in the left ventricular cavity in 10 patients ( 29 injections). The apex, anterolateral wall, and interventricular septum were noted as the sites of contrast efflux into the left ventricular cavity. Simultaneous multiple outflow jets (two or more) were observed in nine patients. In all patients, except the two with hypertrophic cardiomyopathy, contrast appeared within the left ventricle during end systole. In the two patients with hypertrophic cardiomyopathy, contrast was noted in the left ventricular cavity during early systole. Contrast was always noted in the left ventricular cavity subsequent to its appearance in the myocardium, and it always disappeared from the left ventricular cavity after it cleared from the myocardium. Fig. 1 shows an example of two contrast jets in the left ventricular cavity in a patient with coronary artery disease. There was no relation between the presence of prior infarction, site of the coronary artery lesion, or left

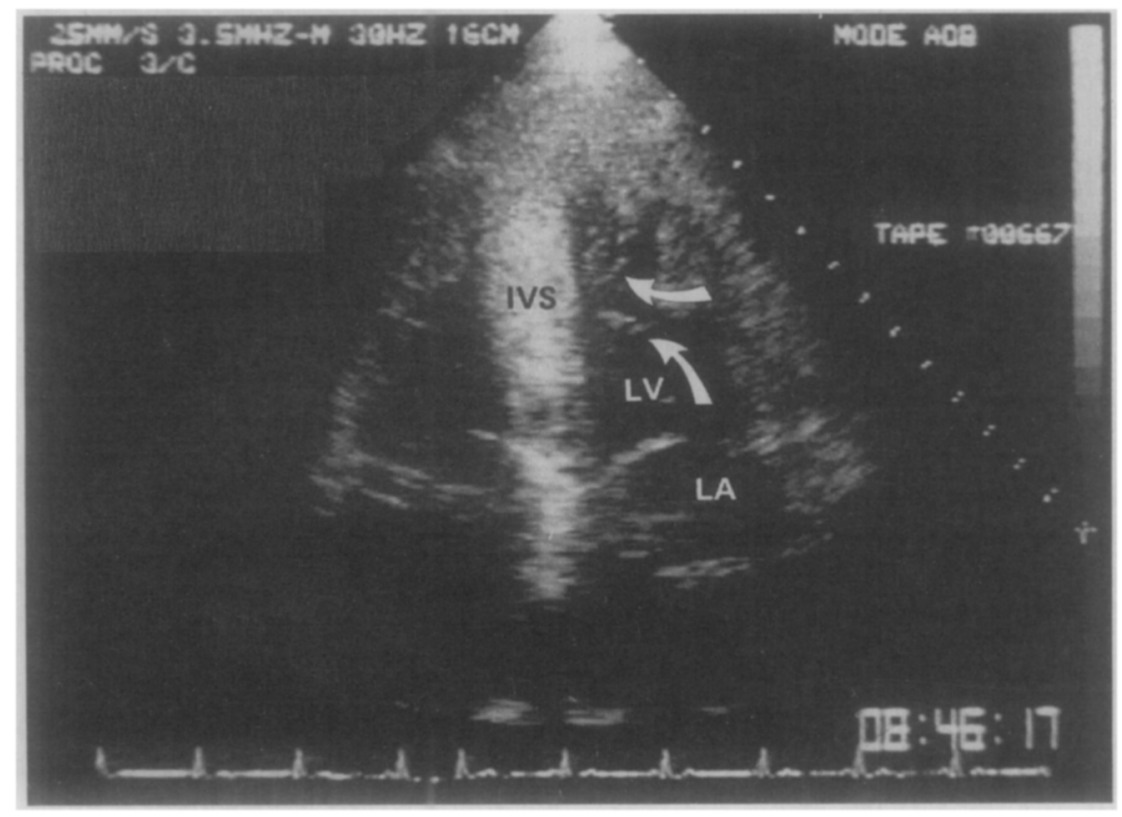

Fig. 1. Example of contrast noted in the left ventricular cavity (arrows), appearing at two sites from the endocardial surface. Note that at this time myocardial opacification is still present. IVS, Interventricular septum; $L A$, left atrium; $L V$, left ventricle. 
ventricular ejection fraction and the appearance of contrast in the left ventricular cavity.

At present, there is no in vivo technique for visualizing venous myocardial drainage in humans. This study reports the potential value of MCE for the visualization of venous drainage from the left ventricular myocardium directly into the left ventricular cavity in patients undergoing coronary arteriography. This finding most likely represents thebesian vein outflow. ${ }^{3}$ The other possibilities include the presence of fistulas or leakage of contrast into the left ventricular cavity from the aortic root. The former is unlikely, since we did not see any fistulas during coronary arteriography. The second possibility is also remote, since we noted the jets emanating directly from the endocardial border of the heart as far from the aortic root as the apex. In this study, we obtained optimal images of the left ventricular cavity and did not always include the right ventricular cavity within the sector. We therefore cannot comment on thebesian vein outflow into the right ventricular cavity. Because the microbubbles used during MCE are about the size of red blood cells, ${ }^{2}$ this might explain the ability of this technique to visualize thebesian vein outflow in humans. Thus MCE has the potential to allow the study of coronary venous drainage in man.

\section{REFERENCES}

1. Tei C, Sakamaki T, Shah PM, et al. Myocardial contrast echocardiography: a reproducible technique of myocardial opacification for identifying regional perfusion deficits. Circulation 1983;67:585-93.

2. Feinstein SB, Ten Cate FJ, Zwehl W, et al. Two-dimensional contrast echocardiography. I. In vitro development and quantitative analysis of echo contrast agents. J Am Coll Cardiol 1984;3:14-20.

3. Hood WB Jr. Regional venous drainage of the human heart. Br Heart J 1968;30:105-9.

\section{Angiographic and intracoronary flow velocity reversal in a transplanted heart: A common etiology for an uncommon condition}

Thomas Donohue, MD, Frank Aguirre, MD, Leslie Miller, MD, and Morton J. Kern, MD. St. Louis, Mo.

Cardiac transplantation is now routinely performed to enhance survival and improve the quality of life for patients with end-stage congestive heart failure. Once accepted as a candidate for heart transplant, these patients must be maintained with aggressive medical management and oc-

From the Cardiology Division, St. Louis University Hospital.

Reprint requests: Morton J. Kern, MD, Cardiac Catheterization Laboratory, St. Louis University Hospital, 3635 Vista Ave, at Grand Blvd., St. Louis, MO 63110-0250.

4/4/35973 casionally they require mechanical assistance while they await a donor heart. Patients who require intravenous inotropic drugs or mechanical assist devices are considered urgent cases and obtain priority on a transplantation list. Despite their severe medical conditions, these patients must await donor hearts that meet certain criteria, including the absence of preexisting coronary or myopathic disease. We present a patient who underwent orthotopic heart transplantation after a prolonged pretransplantation waiting period because of high titer circulating antibodies that created difficulty in obtaining a suitably matched donor heart. This unusual circumstance led to the use of a donor heart with an unusual angiographic and coronary flow velocity pattern observed in the 1-year posttransplantation follow-up angiographic evaluation.

The patient was a 54 -year-old white man admitted for his first annual cardiac catheterization and angiographic evaluation 1 year after orthotopic heart transplantation. Heart transplantation was performed for severe ischemic cardiomyopathy. The pretransplantation period was notable for the acute closure of the patient's own patent native coronary or graft vessels, resulting in the development of cardiogenic shock. This required the placement of a totally implantable pulsatile left ventricular assist device (Novocor, Oakland, Calif.), which functioned well for 9 months before transplantation. ${ }^{1}$ After device insertion and transfusions, he developed a high titer circulating antibody, unfortunately against common antigens, and 9 months went by before a suitable donor heart was found that did not possess the antigens. Using a donor heart that carried the specific antigens would have resulted in hyperacute rejection and death. The patient had no current complaints of chest pain or dyspnea, and his exercise tolerance was excellent. The physical examination was normal, with well-healed surgical scars on the chest and abdomen. At the 1-year study, right heart catheterization revealed normal pressures and cardiac output. Left heart pressures and ventriculography of both chambers were also normal. Coronary arteriography revealed that the dominant right coronary artery was free of disease. The left main and circumflex arteries were also angiographically normal. However, during left coronary angiography, the mid left anterior descending artery was narrowed with a $20 \%$ to $30 \%$ stenosis and demonstrated competitive and reciprocating flow in the midportion. There was no visualization of the distal left anterior descending artery with either left or right coronary injections (Fig. 1). An anomalous left anterior descending coronary ostium could not be found. Severe myocardial coronary artery bridging was considered. To demonstrate the phasic nature of the coronary flow pattern in the left anterior descending artery, an 0.018-inch Doppler-tipped angioplasty guide wire (Cardiometrics, Inc., Mountain View, Calif. $)^{2}$ was advanced through the $6 F$ left Judkins diagnostic catheter. Doppler flow velocities (summarized in Table I) were obtained at several locations along the artery (Fig. 2). The proximal and mid left anterior descending artery Doppler flow patterns demonstrated typical coronary flow velocity (Fig. 3, panels $a$ and $b$ ), with a diminished systolic flow coupled with a predominant larger and longer diastolic flow pattern. When the tip of the Doppler guide 